\title{
OPTIMAL GUARANTEED COST FILTERING FOR MARKOVIAN JUMP DISCRETE-TIME SYSTEMS
}

\author{
MAGDI S. MAHMOUD AND PENG SHI
}

Received 20 August 2001 and in revised form 7 November 2003

This paper develops a result on the design of robust steady-state estimator for a class of uncertain discrete-time systems with Markovian jump parameters. This result extends the steady-state Kalman filter to the case of norm-bounded time-varying uncertainties in the state and measurement equations as well as jumping parameters. We derive a linear state estimator such that the estimation-error covariance is guaranteed to lie within a certain bound for all admissible uncertainties. The solution is given in terms of a family of linear matrix inequalities (LMIs). A numerical example is included to illustrate the theory.

\section{Introduction}

Perhaps the problem of optimal (state and/or parameter) estimation is the oldest problem in systems theory and particularly for dynamical systems subject to stationary Gaussian input and measurement noise processes [1]. For classes of continuous-time and discretetime systems with uncertain parameters, the robust state estimation problem arises naturally for which several techniques have been developed (see $[3,17,20,22,26,30,31]$ and the references cited therein).

Recently, dynamical systems with Markovian jumping parameters have received increasing interests from both control and filtering points of view. For some representative prior work on this general topic, we refer the reader to $[7,8,9,10,11,23,24,25]$ and the references therein. The filtering problem of systems with jumping parameters has been resolved in [11] and a discrete-time filtering problem for hybrid systems has been studied in [12]. In these two papers, the state process is observed in white noise and the random jump process is observed by a point process. The so-called $H_{\infty}$ filter of jump systems has been designed in [9] via a linear matrix inequality (LMI) approach, which provides mean square stable error dynamics and a prescribed bound on the $\mathscr{L}_{2}$-induced gain from the noise signals to the estimation error. The problem of robust Kalman filtering for uncertain linear continuous-time systems with Markovian jump parameters has been studied in [24] in which a state estimator is designed such that the covariance of the estimation 
error is guaranteed to be within a certain bound for all admissible uncertainties. However, to date the problem of robust Kalman filtering for uncertain discrete-time linear systems with Markovian jump parameters, to the best of the authors' knowledge, has not yet been fully investigated.

In this paper, the problem of robust state estimation for linear discrete-time systems with both Markovian jump parameters and norm-bounded parametric uncertainties is investigated. The state estimator is designed such that the estimation-error covariance is guaranteed to be upper bounded for all admissible uncertainties. Our study illustrates that the above problem is solvable if a set of LMIs has a solution. Furthermore, it is shown that the results obtained in this paper encompass the available results in the literature. A numerical example is included to demonstrate the potential of the proposed techniques.

Notations and facts. In the sequel, we denote by $W^{t}, W^{-1}$, and $\lambda(W)$ the transpose, the inverse, and the eigenvalues of any square matrix $W$, respectively. Let $\lambda_{m}(W)$ and $\lambda_{M}(W)$ be the minimum and maximum eigenvalues of matrix $W$. We use $W>0(\geq,<, \leq 0)$ to denote a symmetric positive definite (positive semidefinite, negative, negative semidefinite) matrix and $I$ to denote the $n \times n$ identity matrix, $\mathbb{E}[\cdot]$ stands for mathematical expectation, $\operatorname{tr}(\cdot)$ denotes the matrix trace, and $\ell_{2}[0, \infty]$ is the space of square-summable vectors defined by $\sum_{k=1}^{\infty} f_{k}^{t} f_{k}<\infty$ for $f=\left(f_{k}\right) \in \ell_{2}[0, \infty]$. The symbol $\bullet$ will be used in some matrix expressions to induce a symmetric structure, that is, given matrices $L=L^{t}$ and $R=R^{t}$ of appropriate dimensions, then

$$
\left[\begin{array}{cc}
L+M+\bullet & \bullet \\
N & R
\end{array}\right]=\left[\begin{array}{cc}
L+M+M^{t} & N^{t} \\
N & R
\end{array}\right] .
$$

Also, the notation $(\Omega, \mathscr{F}, \mathbf{P})$ stands for a given probability space, where $\Omega$ is the sample space, $\mathscr{F}$ is the algebra of events, and $\mathbf{P}$ is the probability measure defined on $\mathscr{F}$.

Sometimes the arguments of a function will be omitted in the analysis when no confusion can arise.

Fact 1. For any real matrices $\Sigma_{1}, \Sigma_{2}$, and $\Sigma_{3}$ with appropriate dimensions and $\Sigma_{3}^{t} \Sigma_{3} \leq I$, it follows that

$$
\Sigma_{1} \Sigma_{3} \Sigma_{2}+\Sigma_{2}^{t} \Sigma_{3}^{t} \Sigma_{1}^{t} \leq \alpha \Sigma_{1} \Sigma_{1}^{t}+\alpha^{-1} \Sigma_{2}^{t} \Sigma_{2} \quad \forall \alpha>0
$$

Fact 2. Let $\Sigma_{1}, \Sigma_{2}, \Sigma_{3}$, and $0<R=R^{t}$ be real constant matrices of compatible dimensions and $H(t)$ a real matrix function satisfying $H^{t}(t) H(t) \leq I$. Then, for any $\rho>0$ satisfying $\rho \Sigma_{2}^{t} \Sigma_{2}<R$, the following matrix inequality holds:

$$
\left(\Sigma_{3}+\Sigma_{1} H(t) \Sigma_{2}\right) R^{-1}\left(\Sigma_{3}^{t}+\Sigma_{2}^{t} H^{t}(t) \Sigma_{1}^{t}\right) \leq \rho^{-1} \Sigma_{1} \Sigma_{1}^{t}+\Sigma_{3}\left(R-\rho \Sigma_{2}^{t} \Sigma_{2}\right)^{-1} \Sigma_{3}^{t} .
$$




\section{Discrete-time jumping system}

2.1. Model description. We consider the following class of discrete-time systems with Markovian jump parameters for a given probability space $(\Omega, \mathscr{F}, \mathbf{P})$ :

$$
\begin{gathered}
x_{k+1}=\left[A\left(\eta_{k}\right)+\Delta A\left(k, \eta_{k}\right)\right] x_{k}+w_{k}, \quad x_{0}=\phi, \eta_{0}=i, \\
z_{k}=C\left(\eta_{k}\right) x_{k}+v_{k}, \\
\Delta A\left(k, \eta_{k}\right)=H\left(\eta_{k}\right) \Delta\left(k, \eta_{k}\right) E\left(\eta_{k}\right), \quad\left\|\Delta\left(k, \eta_{k}\right)\right\| \leq 1, k \in \mathscr{L},
\end{gathered}
$$

where $x_{k} \in \mathbb{R}^{n}$ is the system state, $z_{k} \in \mathbb{R}^{p}$ is the system measurement, and $w_{k} \in \mathbb{R}^{n}$ and $v_{k} \in \mathbb{R}^{p}$ are zero-mean Gaussian white-noise processes with joint covariance

$$
\mathbb{E}\left\{\left[\begin{array}{l}
w_{k} \\
v_{k}
\end{array}\right]\left[\begin{array}{l}
w_{k} \\
v_{k}
\end{array}\right]^{t}\right\}=\left[\begin{array}{cc}
\mathcal{W} & 0 \\
0 & \mathscr{V}
\end{array}\right]>0
$$

The initial condition $x_{o}$ is assumed to be a zero-mean Gaussian random variable independent of the white-noise processes $w_{k}$ and $v_{k}$.

The matrices $A\left(\eta_{k}\right) \in \mathbb{R}^{n \times n}$ and $C\left(\eta_{k}\right) \in \mathbb{R}^{p \times n}$ are known real-valued matrices. These matrices are functions of the random process $\left\{\eta_{k}\right\}$ which is a discrete-time, discrete-state Markovian chain taking values in a finite set $\mathscr{Y}=\{1,2, \ldots, s\}$ with generator $\mathfrak{I}=\left(\alpha_{i j}\right)$ and transition probability from mode $i$ at time $k$ to mode $j$ at time $j+1, k \in \mathscr{Y}$ :

$$
p_{i j}=\operatorname{Pr}\left(\eta_{k+1}=j \mid \eta_{k}=i\right)
$$

with $p_{i j} \geq 0$ for $i, j \in \mathscr{S}$ and $\sum_{j=1}^{s} p_{i j}=1$. We note that the set $\mathscr{S}$ consists of different operation modes of system (2.1), and for each value $\eta_{k}=i, i \in \mathscr{Y}$, we denote the matrices associated with mode $i$ by

$$
A\left(\eta_{k}\right)=A_{i}, \quad C\left(\eta_{k}\right)=C_{i},
$$

where $A_{i}$ and $C_{i}$ are known constant matrices describing the nominal system. For $\eta=i \in$ $\mathscr{S}, \Delta A\left(k, \eta_{k}\right)=\Delta A_{i}(k)$ are unknown matrices which represent time-varying uncertainties, and are assumed to belong to certain bounded compact sets, where, for $\eta_{k}=i, i \in \mathscr{Y}$, $H\left(\eta_{k}\right)=H_{i} \in \mathbb{R}^{n \times i}$ and $E\left(\eta_{k}\right)=E_{i} \in \mathbb{R}^{j \times n}$ are known real constant matrices characterizing the way the uncertain parameters $\Delta\left(k, \eta_{k}\right)=\Delta_{i}(k) \in \mathbb{R}^{i \times j}$ affect the nominal matrices $A_{i}$ and $C_{i}$, and $\Delta_{i}(k), i \in \mathscr{S}$, is an unknown time-varying matrix function satisfying (2.1c).

Remark 2.1. Note that system (2.1) can be used to represent many important physical systems subject to random failures and structure changes, such as electric-power systems [28], control systems of a solar thermal central receiver [27], communications systems [2], aircraft flight control [18], control of nuclear power plants [21], and manufacturing systems $[4,5]$. 
2.2. Stochastic quadratic stability. First, we recall the following definition.

Definition 2.2. System (2.1) with $v_{k} \equiv 0, w_{k} \equiv 0$, and $\Delta_{i}(k) \equiv 0$ is said to be stochastically stable (SS) if for all finite initial state $\phi \in \mathbb{R}^{n}$ and initial mode $\eta_{o} \in \mathscr{Y}$, there exists a finite number $\mathcal{N}\left(\phi, \eta_{0}\right)>0$ such that

$$
\lim _{R \rightarrow \infty} \mathbb{E}\left[\sum_{k=0}^{R} x_{k}^{t}\left(\phi, \eta_{o}\right) x_{k}\left(\phi, \eta_{o}\right) \mid \phi, \eta_{o}\right]<\mathcal{N}\left(\phi, \eta_{o}\right)
$$

Remark 2.3. In light of $[6,13]$, it follows that $(2.5)$ is equivalent to mean square stability (MSS) in the sense that

$$
\lim _{k \rightarrow \infty} \mathbb{E}\left[\left\|x_{k}\right\| \mid \phi, \eta_{o}\right] \longrightarrow 0
$$

and, in turn, it implies almost sure stability (ASS) in the sense that, for every finite initial state $\phi \in \mathbb{R}^{n}$ and initial mode $\eta_{o} \in \mathscr{Y}$, we have

$$
\lim _{k \rightarrow \infty} x(k) \longrightarrow 0
$$

with probability 1 .

Lemma 2.4. System (2.1) with $v_{k} \equiv 0, w_{k} \equiv 0$, and $F\left(k, \eta_{k}\right) \equiv 0$ is SS if and only if there exists a set of matrices $\left\{W_{i}=W_{i}^{t}>0\right\}, i \in \mathscr{Y}$, satisfying the following set of coupled LMIs:

$$
A_{i}^{t}\left[\sum_{j=1}^{s} p_{i j} W_{j}\right] A_{i}-W_{i}<0, \quad i=1, \ldots, s .
$$

Proof. Let the modes at times $k$ and $k+1$ be $\eta_{k}=i, \eta_{k+1}=j \in \mathscr{Y}$. Take the stochastic Lyapunov function candidate $V(\cdot)$ to be (see [14])

$$
V_{k}\left(x_{k}, i\right)=x_{k}^{t} W(i) x_{k}
$$

Thus, we have from (2.9), together with (2.8),

$$
\begin{aligned}
\mathbb{E}\left\{V_{k+1}\left(x_{k+1}, j\right) \mid x_{k}, i\right\}-V_{k}\left(x_{k}, i\right) & =\sum_{j=1}^{s} p_{i j} x_{k+1}^{t} W_{j} x_{k+1}-x_{k}^{t} W_{i} x_{k} \\
& =\sum_{j=1}^{s} p_{i j} x_{k}^{t} A_{i}^{t} W_{j} A_{i} x_{k}-x_{k}^{t} W_{i} x_{k} \\
& =-x_{k}^{t} Q_{i} x_{k}<0 .
\end{aligned}
$$


With $Q_{i}>0$, we have from (2.10), for $x_{k} \neq 0$,

$$
\frac{\mathbb{E}\left\{V_{k+1}\left(x_{k+1}, j\right) \mid x_{k}, \eta_{k}\right\}-V_{k}\left(x_{k}, i\right)}{V_{k}\left(x_{k}, i\right)}<-\frac{-x_{k}^{t} Q_{i} x_{k}}{x_{k}^{t} W(i) x_{k}} \leq-\min _{i \in \mathscr{S}}\left\{\frac{\lambda_{m}\left(-Q_{i}\right)}{\lambda_{M}\left(P_{i}\right)}\right\}=\beta-1,
$$

where

$$
\beta=1-\min _{i \in \mathscr{S}}\left\{\frac{\lambda_{m}\left(-Q_{i}\right)}{\lambda_{M}\left(P_{i}\right)}\right\}
$$

Since

$$
\beta>\frac{\mathbb{E}\left\{V_{k+1}\left(x_{k+1}, j\right) \mid x_{k}, \eta_{k}\right\}}{V_{k}\left(x_{k}, i\right)}=\frac{\sum_{j=1}^{s} p(i j)\left(x_{k+1}^{t} W_{j} x_{k+1}\right)}{V_{k}\left(x_{k}, i\right)}>0,
$$

and in view of (2.11), it is readily evident that $0<\beta<1$, and hence

$$
\mathbb{E}\left\{V_{k+1}\left(x_{k+1}, j\right) \mid x_{k}, i\right\}<\beta V_{k}\left(x_{k}, i\right) \Longrightarrow \mathbb{E}\left\{V_{k}\left(x_{k}, i\right) \mid \phi, \eta_{o}\right\}<\beta^{k} V_{0}\left(\phi, \eta_{o}\right)
$$

It follows from (2.14) that

$$
\mathbb{E}\left[\sum_{k=0}^{R} V_{k}\left(x_{k}, \eta_{k}\right) \mid \phi, \eta_{0}\right]<\left(1+\beta+\cdots+\beta^{R}\right) V_{0}\left(\phi, \eta_{o}\right)=\frac{1-\beta^{R+1}}{1-\beta} V_{0}\left(\phi, \eta_{o}\right),
$$

and hence

$$
\lim _{R \rightarrow \infty} \mathbb{E}\left[\sum_{k=0}^{R} x_{k}^{t} W\left(\eta_{k}\right) x_{k} \mid \phi, \eta_{o}\right]<\frac{1}{1-\beta} V_{0}\left(\phi, \eta_{o}\right)
$$

Introducing

$$
\mathcal{N}\left(\phi, \eta_{o}\right) \triangleq \frac{\max _{i \in \mathscr{S}}\left\{W_{i}^{-1}\right\}}{1-\beta} V_{0}\left(\phi, \eta_{o}\right)
$$

and using Rayleigh quotient, we have

$$
\lim _{R \rightarrow \infty} \mathbb{E}\left[\sum_{k=0}^{R} x_{k}^{t} x_{k} \mid \phi, \eta_{o}\right]=\lim _{R \rightarrow \infty} \mathbb{E}\left[\sum_{k=0}^{R}\left\|x_{k}\right\|^{2} \mid \phi, \eta_{o}\right]<\mathcal{N}\left(\phi, \eta_{o}\right),
$$

which means that system (2.1) is SS, thus the sufficiency part is proved. The proof of necessity can be found in [13]. 
Remark 2.5. Lemma 2.4 establishes an LMI stability test of the input-free nominal jump system. It is easy to show that $(2.8)$ is equivalent to the fact that there exists a set of matrices $\left\{Z_{i}>0, i \in \mathscr{S}\right\}$ satisfying

$$
A_{i}^{t}\left[\sum_{j=1}^{s} p_{i j} Z_{j}\right] A_{i}-Z_{i}=0, \quad i=1, \ldots, s .
$$

In line with the results of [15] for linear systems, we introduce the following definition of stochastic quadratic stability.

Definition 2.6. System (2.1) with $w_{k} \equiv 0$ is said to be stochastically quadratically stable if there exists a set of matrices $\left\{0<W_{i}=W_{i}^{t}, i \in \mathscr{Y}\right\}$ satisfying

$$
\left[A_{i}+\Delta A_{i}(k)\right]^{t}\left[\sum_{j=1}^{s} p_{i j} W_{j}\right]\left[A_{i}+\Delta A_{i}(k)\right]-W_{i}<0, \quad i \in \mathscr{S}
$$

for all admissible parameter uncertainties $\Delta A_{i}(k), i \in \mathscr{Y}$, satisfying (2.1c).

Now, we show that for system (2.1), stochastic quadratic stability implies stochastic stability.

THEOREM 2.7. System (2.1) with $w_{k} \equiv 0$ is SS for all admissible parameter uncertainties $\Delta A_{i}(k), i \in \mathscr{Y}$, if it is stochastically quadratically stable.

Proof. Since system (2.1) with $w_{k} \equiv 0$ is stochastically quadratically stable, by Definition 2.6, there exists a set of matrices $\left\{0<W_{i}=W_{i}^{t}, i \in \mathscr{Y}\right\}$ satisfying (2.20) for all admissible parameter uncertainties $\Delta A_{i}(k), i \in \mathscr{Y}$; thus (2.1a) is SS.

By direct application of Fact 2 and rearranging terms, we have the following corollary. Corollary 2.8. System (2.1) with $w_{k} \equiv 0$ is SS for all admissible parameter uncertainties $\Delta A_{i}(k), i \in \mathscr{Y}$, if there exist a set of matrices $\left\{W_{i}=W_{i}^{t}>0\right\}, i \in \mathscr{Y}$, and a set of scalars $\rho_{i}>0, i \in \mathscr{Y}$, satisfying the following set of coupled LMIs:

$$
\rho_{i}^{-1} H_{i} H_{i}^{t}+A_{i}\left[\bar{W}_{i}^{-1}-\rho_{i} E_{i}^{t} E_{i}\right]^{-1} A_{i}^{t}-W_{i}<0,
$$

where

$$
\bar{W}_{i}=\sum_{j=1}^{s} p_{i j} W_{j}, \quad i \in \mathscr{S} .
$$

2.3. State estimator. Our purpose in this paper is to design a state estimator of the form

$$
\hat{x}_{k+1}=F_{i} \hat{x}_{k}+G_{i} z_{k}
$$


for $i \in \mathscr{Y}$, where $\hat{x}_{k} \in \mathbb{R}^{n}$ is the state estimate and $\hat{x}_{k_{o}}$ is the estimator initial condition which is assumed to be a zero-mean Gaussian random vector. The matrices $G_{i}$ and $K_{i}$, $i \in \mathscr{Y}$, are the estimator gain to be determined in order that the estimation error dynamics be stochastically asymptotically stable. When such an estimator is applied to the uncertain system (2.1), the corresponding estimation error vector is defined by $e_{k}=x_{k}-\hat{x}_{k}$. From (2.1) and estimator (2.23), for $\eta_{k}=i$, one has

$$
e_{k+1}=F_{i} e_{k}+\left[A_{i}-F_{i}-G_{i} C_{i}\right] x_{k}+\Delta A(k, i) x_{k}+w_{k}-G_{i} v_{k}
$$

In terms of the state variables $e_{k}$ and $\hat{x}_{k}$, the state equations describing the augmented system obtained from (2.1) and (2.24) are as follows:

$$
\xi_{k+1}=\left[\bar{A}_{i}+\bar{H}_{i} \Delta_{i}(k) \bar{E}_{i}\right] \xi_{k}+\bar{B}_{i} \sigma_{k}, \quad \xi_{k_{o}}=\xi_{0}
$$

where

$$
\begin{aligned}
& \xi_{k}=\left[\begin{array}{c}
e_{k} \\
\hat{x}_{k}
\end{array}\right], \quad \xi_{k_{o}}=\left[\begin{array}{c}
x_{k_{o}}-\hat{x}_{k_{o}} \\
\hat{x}_{k_{o}}
\end{array}\right], \quad \sigma_{k}=\left[\begin{array}{c}
\mathcal{W}^{-1 / 2} w_{k} \\
\mathcal{V}^{-1 / 2} v_{k}
\end{array}\right], \\
& \bar{A}_{i}=\left[\begin{array}{cc}
A_{i}-G_{i} C_{i} & A_{i}-F_{i}-G_{i} C_{i} \\
G_{i} C_{i} & F_{i}+G_{i} C_{i}
\end{array}\right], \quad \bar{H}_{i}=\left[\begin{array}{c}
H_{i} \\
0
\end{array}\right], \\
& \bar{E}_{i}=\left[\begin{array}{ll}
E_{i} & E_{i}
\end{array}\right], \quad \bar{B}_{i}=\left[\begin{array}{cc}
\mathcal{W}^{1 / 2} & -G_{i} \mathscr{V}^{1 / 2} \\
0 & G_{i} \mathscr{V}^{1 / 2}
\end{array}\right] .
\end{aligned}
$$

We introduce the following definition.

Definition 2.9. The state estimator (2.23) is said to be an SS quadratic guaranteed cost (SSQGC) state estimator with associated set of cost matrices $\left\{0<X_{i}=X_{i}^{t}, i \in \mathscr{Y}\right\}$ for system (2.1) if for the estimator gain matrix $F,|\lambda(F)|<1$, and there exist matrices $\mathscr{X}_{i}$, $i \in \mathscr{S}$, satisfying

$$
\mathscr{X}_{i}=\left[\begin{array}{cc}
X_{i} & \Pi_{i} \\
\Pi_{i}^{t} & Y_{i}
\end{array}\right]
$$

such that the inequality

$$
\left[\bar{A}_{i}+\bar{H}_{i} \Delta(k, i) \bar{E}_{i}\right] \overline{\mathscr{X}}_{i}\left[\bar{A}_{i}+\bar{H}_{i} \Delta(k, i) \bar{E}_{i}\right]^{t}-\mathscr{X}_{i}+\bar{B}_{i} \bar{B}_{i}^{t}<0
$$

holds for all uncertainties $\|\Delta(k, i)\| \leq 1$, where $\overline{\mathscr{X}}_{i}=\sum_{j=1}^{s} p_{i j} X_{j}, i \in \mathscr{Y}$.

In the discussions to follow, we restrict attention to the class of quadratic guaranteed cost state estimators. The next result shows that if estimator (2.23) is an SSQGC for system (2.1) with cost matrix $X_{i}, i \in \mathscr{Y}$, then $X_{i}$ provides an upper bound on the steady-state error covariance matrix at time $k$ :

$$
X_{i c}(k)=\lim _{k_{o} \rightarrow \infty} \mathbb{E}\left\{e_{k}^{t} e_{k}\right\}
$$


Theorem 2.10. Suppose that (2.23) is an SSQGC state estimator with cost matrix $X_{i}, i \in \mathscr{Y}$, for system (2.1). Then the augmented system (2.25) will be stochastically quadratically stable and the steady-state error covariance matrix at time $k$ satisfies the bound

$$
X_{i c}(k) \leq X_{i}(k), \quad i \in \mathscr{Y}
$$

for all admissible uncertainties $\Delta(k, i)$.

Conversely, any state estimator of type (2.23) with $|\lambda(F)|<1$ will be an SSQGC state estimator for system (2.1) with some cost matrix $\tilde{X}_{i}>0$.

Proof

Necessity. Suppose that (2.23) is an SSQGC state estimator for system (2.1) with cost matrix $X_{i}>0$. Since for the matrix $F,|\lambda(F)|<1$, and (2.1) is stochastically quadratically stable, it follows that the augmented system (2.25) will be stochastically quadratically stable. This can be easily verified on selecting a Lyapunov matrix of the form

$$
\Omega_{i}=\left[\begin{array}{cc}
\omega_{i} \Omega_{i s} & 0 \\
0 & \Omega_{i f}
\end{array}\right]
$$

with $\omega_{i}>0$ being a sufficiently large constant and the matrices $\Omega_{i s}$ and $\Omega_{i f}$ quadratic Lyapunov functions for system (2.1) and (2.23), respectively. Let $\mathbb{E}\left\{\xi_{k_{o}} \xi_{k_{o}}^{t}\right\}=\Xi_{i} \geq 0$. Since $\sigma_{k}$ is a Gaussian white-noise process with identity covariance, it follows for any admissible uncertainty $\Delta(k, i) \leq 1$ that the state covariance matrix for system (2.25) is given by

$$
\hat{X}_{i c}\left(k, k_{o}\right) \triangleq \mathbb{E}\left\{\xi_{k} \xi_{k}^{t}\right\}=\Phi\left(k, k_{o}\right) \Xi_{i} \Phi^{t}\left(k, k_{o}\right)+\sum_{j=k_{o}}^{k} \Phi(k, j) \bar{B}_{i} \bar{B}_{i}^{t} \Phi^{t}(k, j),
$$

where $\Phi(k, j)$ is the state transition matrix associated with system (2.25). Moreover, using the fact that system (2.25) is stochastically quadratically stable, it follows that

$$
\Phi\left(k, k_{o}\right)=\lim _{k_{o} \rightarrow \infty}=0
$$

which in turn implies that

$$
\hat{X}_{i c}(k) \triangleq \lim _{k_{o} \rightarrow \infty} \hat{X}_{i c}\left(k, k_{o}\right)=\sum_{j=k_{o}}^{k} \Phi(k, j) \bar{B}_{i} \bar{B}_{i}^{t} \Phi^{t}(k, j) .
$$

Introducing

$$
\begin{gathered}
\Upsilon_{i}(k) \triangleq \mathscr{X}_{i}-\left[\bar{A}_{i}+\bar{H}_{i} \Delta(k, i) \bar{E}_{i}\right] \overline{\mathscr{X}}_{i}\left[\bar{A}_{i}+\bar{H}_{i} \Delta(k, i) \bar{E}_{i}\right]^{t}-\bar{B}_{i} \bar{B}_{i}^{t}>0, \\
\Lambda_{i}\left(k, k_{o}\right)=\overline{\mathscr{X}}_{i}-\hat{X}_{i c}\left(k, k_{o}\right),
\end{gathered}
$$

it is readily evident that $\Lambda_{i}\left(k, k_{o}\right)$ satisfies the Lyapunov difference equation

$$
\Lambda_{i}\left(k+1, k_{o}\right)=\left[\bar{A}_{i}+\bar{H}_{i} \Delta(k, i) \bar{E}_{i}\right] \Lambda_{i}\left(k, k_{o}\right)\left[\bar{A}_{i}+\bar{H}_{i} \Delta(k, i) \bar{E}_{i}\right]^{t}+\Upsilon_{i}(k),
$$


and hence, in view of the stochastic quadratic stability of system (2.25),

$$
\lim _{k_{o} \rightarrow \infty} \Lambda_{i}\left(k, k_{o}\right)=\overline{\mathscr{d}}_{i}-\hat{X}_{i c}(k)>0, \quad i \in \mathscr{Y}
$$

holds independently of the initial condition $\Lambda_{i}\left(k_{o}, k_{o}\right)$. Thus, $\hat{X}_{i c}(k) \leq \overline{\mathscr{X}}_{i}$ for all admissible uncertainties $\|\Delta(k, i)\| \leq 1$. In view of the matrix structure of (2.27), it follows that

$$
X_{i c}(k) \leq X_{i}
$$

for all admissible uncertainties $\Delta(k, i) \leq 1$ and for all $i \in \mathscr{Y}$.

Sufficiency. Now, consider any state estimator of the form (2.23) with $F,|\lambda(F)|<1$. Again, since system (2.1) is stochastically quadratically stable, it easily follows that the augmented system (2.25) will be stochastically quadratically stable. Therefore, there exists a matrix

$$
\tilde{\mathscr{X}}_{i}=\left[\begin{array}{cc}
\tilde{X}_{i} & \tilde{\Pi}_{i} \\
\tilde{\Pi}_{i}^{t} & \tilde{Y}_{i}
\end{array}\right]
$$

such that

$$
\left[\bar{A}_{i}+\bar{H}_{i} \Delta(k, i) \bar{E}_{i}\right] \tilde{\mathscr{\mathscr { Q }}}_{i}\left[\bar{A}_{i}+\bar{H}_{i} \Delta(k, i) \bar{E}_{i}\right]^{t}-\tilde{\mathscr{Q}}_{i}<0 \quad \forall\|\Delta(k, i)\| \leq 1 .
$$

Hence, there exist constants $\varepsilon_{i}>0, i \in \mathscr{Y}$, such that

$$
\left[\bar{A}_{i}+\bar{H}_{i} \Delta(k, i) \bar{E}_{i}\right] \varepsilon_{i}^{-1} \tilde{\mathscr{\mathscr { X }}}_{i}\left[\bar{A}_{i}+\bar{H}_{i} \Delta(k, i) \bar{E}_{i}\right]^{t}-\varepsilon_{i}^{-1} \tilde{\mathscr{X}}_{i}+\bar{B}_{i} \bar{B}_{i}^{t}<0 \quad \forall\|\Delta(k, i)\| \leq 1 .
$$

We conclude that this estimator is an SSQGC state estimator with cost matrix $\varepsilon_{i}^{-1} \tilde{X}_{i}$.

Remark 2.11. Since our main purpose is to construct a state estimator which minimizes the upper bound on the error covariance $X_{i}, i \in \mathscr{Y}$, we solve an alternative minimization problem by looking at the corresponding bound on the steady-state mean square error

$$
\lim _{k_{o} \rightarrow \infty} \mathbb{E}\left\{e_{k}^{t} e_{k}\right\}=\operatorname{Tr}\left[X_{i c}(k)\right] \leq \operatorname{Tr}\left[X_{i}\right]
$$

We will be concerned with constructing an SSQGC state estimator which minimizes $\operatorname{Tr}\left[X_{i}\right]$. In the case of limited-state measurements, it may be required to estimate an output variable $y_{k}=L x_{k}$. The solution would be an output estimate of the form $\hat{y}_{k}=L x_{k}$ and the corresponding steady-state mean square error bound

$$
\lim _{k_{o} \rightarrow \infty} \mathbb{E}\left\{\left[y_{k}-\hat{y}_{k}\right]^{t}\left[y_{k}-\hat{y}_{k}\right]\right\}=\operatorname{Tr}\left[L^{t} X_{i c}(k) L\right] \leq \operatorname{Tr}\left[L^{t} X_{i} L\right]
$$

This means that an SSQGC state estimator would be constructed to minimize the quantity $\operatorname{Tr}\left[L^{t} X_{i} L\right]$. 


\section{Construction of the optimal filter}

In this section, we provide an LMI approach to constructing the SSQGC state estimator for system (2.1), which minimizes the bound in (2.42). We show that the filtering problem can be solved if a family of coupled LMIs has a feasible solution. For simplicity in exposition, we assume that the matrix $A_{i}$ is invertible for all $i \in \mathscr{Y}$. The following theorem establishes the main result.

Theorem 3.1. Consider system (2.1) and suppose that it is stochastically quadratically stable. If there exist two sets of matrices $\left\{\Phi_{i}=\Phi_{i}^{t}>0, \Psi_{i}=\Psi_{i}^{t}>0, i \in \mathscr{Y}\right\}$ and a set of scalars $\left\{\mu_{i}>0, i \in \mathscr{Y}\right\}$ such that the LMIs

$$
\begin{gathered}
{\left[\begin{array}{cccc}
-\Phi_{i}+\mu_{i} I & A_{i}^{t} \bar{\Phi}_{i} & A_{i}^{t} \bar{\Phi}_{i}{ }^{a} \bar{W}_{i}^{1 / 2} & E_{i}^{t} \\
\bullet & -\bar{\Phi}_{i} & 0 & 0 \\
\bullet & \bullet & -I+\overline{\mathcal{W}}_{i}^{1 / 2} \bar{\Phi}_{i} \bar{W}_{i}^{1 / 2} & 0 \\
\bullet & \bullet & \bullet & -\epsilon_{i} I
\end{array}\right]<0,} \\
{\left[\begin{array}{cc}
-\Psi_{i}+M_{i}+\Upsilon_{i} & \hat{A}_{i} \bar{\Psi}_{i} \\
\bullet & -\bar{\Psi}_{i}
\end{array}\right]<0, \quad\left[\begin{array}{cc}
\Upsilon_{i} & \hat{A}_{i} \bar{\Psi}_{i} \hat{C}_{i}^{t}+L_{i} \\
\bullet & -\hat{R}_{i}-\hat{C}_{i} \bar{\Psi}_{i} \hat{C}_{i}^{t}
\end{array}\right]>0}
\end{gathered}
$$

have a feasible solution, where

$$
\begin{gathered}
\bar{\Phi}_{i}=\sum_{j=1}^{s} p_{i j} \Phi_{j}, \quad \bar{\Psi}_{i}=\sum_{j=1}^{s} p_{i j} \Psi_{j}, \\
\bar{W}_{i}=\mathscr{W}+\epsilon_{i} H_{1 i} H_{1 i}^{t}, \\
I-\bar{W}_{i}^{1 / 2} \bar{\Phi}_{i} \bar{W}^{1 / 2}>0, \\
\hat{A}_{i}=A_{i}+\delta A_{i}=A_{i}+\overline{\mathcal{W}}_{i}\left[\bar{\Phi}_{i}^{-1}-\overline{\mathcal{W}}_{i}\right]^{-1} A_{i}, \\
\hat{C}_{i}=C_{i}+\delta C_{i}=C_{i}+\epsilon_{i}^{-1} H_{2 i} H_{1 i}^{t}\left[\bar{\Phi}_{i}^{-1}-\overline{\mathcal{W}}_{i}\right]^{-1} A_{i}, \\
\hat{R}_{i}=\mathscr{V}+\epsilon_{i}^{-1} H_{2 i} H_{2 i}^{t}+\epsilon_{i}^{-2} H_{2 i} H_{1 i}^{t}\left[\Phi_{i}^{-1}-\bar{W}_{i}\right]^{-1} H_{1 i} H_{2 i}^{t}, \\
L_{i}=\epsilon_{i}^{-1}\left[I-\overline{\mathcal{W}}_{i} \bar{\Phi}_{i}^{-1}\right]^{-1} H_{1 i} H_{2 i}^{t}, \\
M_{i}=\overline{\mathcal{W}}_{i}+\overline{\mathcal{W}}_{i}\left[\bar{\Phi}_{i}^{-1}-{ }_{\mathcal{W}} \overline{\mathcal{W}}_{i}\right]^{-1} \overline{\mathcal{W}}_{i},
\end{gathered}
$$

then the estimator (2.23) is an SSQGC state estimator with gains

$$
G_{i}=\left[\hat{A}_{i} \bar{\Psi}_{i} \hat{C}_{i}^{t}+L_{i}\right]\left(\hat{R}_{i}+\hat{C}_{i} \bar{\Psi}_{i} \hat{C}_{i}^{t}\right)^{-1}, \quad F_{i}=\hat{A}_{i}-G_{i} \hat{C}_{i},
$$

with guaranteed cost

$$
\mathbb{E}\left\{[x(k)-\hat{x}(k)]^{t}[x(k)-\hat{x}(k)]\right\} \leq \sigma_{i} \triangleq \max _{i \in \mathscr{S}} \operatorname{tr}\left(\Psi_{i}\right) .
$$


Proof. The proof essentially follows a line similar to the proof of a result in the work of Xie et al. [32]. First, in view of the stochastic quadratic stability of system (2.1a), it follows from the results of $[15,32]$ that, for each fixed $i \in \mathscr{Y}$,

$$
\left\|E_{i}\left(z I-A_{i}\right)^{-1} H_{1 i}\right\|_{\infty}<1
$$

For an arbitrary small $\nu>0$ and a sufficiently small $\epsilon_{i}>0$, inequality (3.13) implies that

$$
\left\|\left[E_{i}^{t} \nu^{1 / 2} I\right]^{t}\left(z I-A_{i}\right)^{-1}\left[H_{1 i} \epsilon_{i}^{1 / 2} W^{1 / 2}\right]\right\|_{\infty}<1
$$

By the discrete bounded real lemma $[16,19]$, there exists a matrix $\Xi_{i}=\Xi_{i}^{t}>0$ with $\bar{\Xi}_{i}=$ $\sum_{j=1}^{s} p_{i j} \Xi_{j}$ satisfying $\bar{\Xi}_{i}^{-1}-\epsilon_{i} W-H_{1 i} H_{1 i}^{t}>0$ such that

$$
A_{i}^{t}\left[\bar{\Xi}_{i}^{-1}-\epsilon_{i}{ }^{W}-H_{1 i} H_{1 i}^{t}\right]^{-1} A_{i}-\Xi_{i}+E_{i}^{t} E_{i}+\nu I<0 .
$$

Letting $\Xi_{i}^{*}=\epsilon_{i} \Xi_{i}$ with $\bar{\Xi}_{i}^{*}=\sum_{j=1}^{s} p_{i j} \Xi_{j}^{*}$ and $\mu_{i}=\epsilon_{i} \nu$, using (3.4) and applying the matrixinversion lemma [16], it follows from (3.15) that

$$
A_{i}^{t} \bar{\Xi}_{i}^{*} A_{i}-\Xi_{i}^{*}+A_{i}^{t} \bar{\Xi}_{i}^{*} \bar{W}_{i}^{1 / 2}\left[I-\overline{W_{i}^{1 / 2}} \bar{\Xi}_{i}^{*} \overline{W_{i}^{1 / 2}}\right]^{-1} \mathcal{W}_{i}^{1 / 2} \bar{\Xi}_{i}^{*} A_{i}+\epsilon_{i} E_{i}^{t} E_{i}+\mu_{i} I<0 .
$$

Inequality (3.16) is feasible provided that $\hat{A}_{i}$ is a stable matrix and $\bar{W}_{i}^{1 / 2} \bar{\Phi}_{i}{ }^{1 / 2}<I$, where $\bar{\Phi}_{i}=\sum_{j=1}^{s} p_{i j} \Phi_{j}$.

Using parallel arguments, it follows from [1] that (3.2) is an LMI for the stationary standard linear filtering, where $M_{i}$ and $\hat{R}_{i}$ are the covariance matrices of the process and measurement noise signals, respectively, and $L_{i}$ is the cross-covariance matrix between the process and measurement noises.

To establish the stochastic quadratic stability of the estimator (2.23), we define

$$
\mathscr{Y}_{i}=\left[\begin{array}{cc}
\Phi_{i}^{-1} & 0 \\
0 & \Psi_{i}
\end{array}\right]
$$

where $\Phi_{i}$ and $\Psi_{i}$ are the feasible solutions to the LMIs (3.1) and (3.2), respectively. In terms of

$$
\mathscr{E}_{i}^{t \in \mathscr{E}} \mathscr{E}_{i}=E_{i}^{t} E_{i}+\nu I, \quad \overline{\mathscr{E}}_{i}=\left[\begin{array}{ll}
\mathscr{E}_{i} & 0
\end{array}\right],
$$

and (2.26), it can be shown by algebraic manipulations that

$$
\bar{A}_{i}^{t} \overline{\mathscr{Y}}_{i} \bar{A}_{i}-\mathscr{Y}_{i}+\epsilon_{i} \bar{A}_{i}^{t} \bar{y}_{i} \overline{\mathscr{C}}_{i}^{t}\left[I-\epsilon_{i} \overline{\mathscr{C}}_{i} \overline{\mathscr{Y}}_{i} \overline{\mathscr{E}}_{i}\right]^{-1} \overline{\mathscr{C}}_{i} \overline{\mathscr{Y}}_{i} \bar{A}_{i}+\epsilon_{i}^{-1} \bar{H}_{i} \bar{H}_{i}^{t}+\bar{B}_{i} \bar{B}_{i}^{t}=0
$$

and $I-\epsilon_{i} \overline{\mathscr{E}}_{i} \overline{\mathscr{Y}}_{i} \overline{\mathscr{E}}_{i}>0$. Observe that $\overline{\mathscr{E}}_{i}^{t} \overline{\mathscr{E}}_{i} \geq \bar{E}_{i}^{t} \bar{E}_{i}$ implies that $I-\epsilon_{i} \bar{E}_{i} \bar{Y}_{i} \bar{E}_{i}>0$ and

$$
\bar{A}_{i}^{t} \overline{\mathscr{Y}}_{i} \bar{A}_{i}-\mathscr{Y}_{i}+\epsilon_{i} \bar{A}_{i}^{t} \overline{\mathscr{Y}}_{i} \bar{E}_{i}^{t}\left[I-\epsilon_{i} \bar{E}_{i} \overline{\mathscr{Y}}_{i} \bar{E}_{i}\right]^{-1} \bar{E}_{i} \overline{\mathscr{Y}}_{i} \bar{A}_{i}+\epsilon_{i}^{-1} \bar{H}_{i} \bar{H}_{i}^{t}+\bar{B}_{i} \bar{B}_{i}^{t} \leq 0 .
$$


For all $\Delta_{i}(k):\left\|\Delta_{i}(k)\right\|<1$, it follows from [16] and Fact 2 that (3.20) leads to

$$
\left[\bar{A}_{i}+\bar{H}_{i} F_{i}(k) \bar{E}_{i}\right] \bar{Y}_{i}\left[\bar{A}_{i}+\bar{H}_{i} F_{i}(k) \bar{E}_{i}\right]^{t}-\mathscr{Y}_{i}+\bar{B}_{i} \bar{B}_{i}^{t} \leq 0,
$$

where $\overline{\mathscr{Y}}_{i}=\sum_{j=1}^{s} p_{i j} \mathscr{y}_{j}$. It follows from Theorem 2.10 that $(2.23)$ is a stochastic stable quadratic estimator with a guaranteed cost given by (3.12).

Remark 3.2. It should be observed that Theorem 3.1 provided an LMI-based feasibility test which can be conveniently solved by Matlab-LMI solver. The matrix $\Upsilon_{i}, i \in \mathscr{Y}$, is an intermediate variable introduced to facilitate the well-posedness of the problem. In this study, it is assumed that jumping parameter information $\left\{\eta_{k}, k=1,2, \ldots\right\}$ is available for our design. However, if it is not the case, then Wonham filtering technique [29] would be required to first estimate the Markov chain observed in Gaussian noise, and the approach presented in this paper can then be employed. Also, it should be noted that the designed state estimator/filter (2.23) depends upon the system mode $i$. This is because due to the existence of the jumping parameters $\left\{\eta_{k}\right\}$ in the system, the "complicated" dependence is unavoidable, otherwise, the filter (independent of $i$ ) would be very conservative (using one operating form for the whole system). Indeed, the dependence is good in the sense that it gives us more options for designing and choosing the better, if not the best, filter to better estimate the system state. That is, if the system has more chance to stay in mode $i$, then the filter (2.23) would be likely to be chosen at $i$ th form. Similarly, the filter can be chosen at $j$ th form if the system is likely to jump from $i$ mode to $j$ mode (with high probability).

Remark 3.3. In effect, the estimation error with minimum covariance of system (2.1) can be determined by the following minimization problem: minimize $\sigma_{i}$ subject to

$$
\epsilon_{i}>0, \quad \Phi_{i}>0, \quad \Psi_{i}>0, \quad i \in \mathscr{Y},
$$

where $\Phi_{i}$ and $\Psi_{i}, i \in \mathscr{Y}$, are the feasible solutions of (3.1) and (3.2).

\section{Example}

In order to illustrate Theorem 3.1, we consider a pilot-scale multireach water-quality system which can fall into the type (2.1a) and (2.1b). Let the Markov process governing the mode switching have the infinitesimal generator (see [29])

$$
\mathfrak{J}=\left[\begin{array}{ccc}
-5 & 2 & 3 \\
1 & -4 & 3 \\
4 & 3 & -7
\end{array}\right]
$$

For the three operating conditions (modes), the associated dates are as follows: 
Mode 1.

$$
\begin{aligned}
A(1) & =\left[\begin{array}{ccc}
0.3 & -0.2 & 0.1 \\
0.02 & 0.5 & 0.1 \\
-0.1 & 0.1 & 0.4
\end{array}\right], \quad C(1)=\left[\begin{array}{ll}
0 & 0 \\
0 & 1 \\
1 & 0
\end{array}\right], \\
H_{1}(1) & =\left[\begin{array}{l}
0.1 \\
0.1 \\
0.1
\end{array}\right], \quad E^{t}(1)=\left[\begin{array}{l}
0.5 \\
0.4 \\
0.2
\end{array}\right] .
\end{aligned}
$$

Mode 2.

$$
\begin{aligned}
A(2) & =\left[\begin{array}{ccc}
-0.4 & 0 & 0.2 \\
0.2 & 0.5 & 0 \\
0 & -0.2 & 0.6
\end{array}\right], \quad C(2)=\left[\begin{array}{ll}
1 & 0 \\
0 & 1 \\
0 & 0
\end{array}\right], \\
H_{1}(2) & =\left[\begin{array}{l}
0.15 \\
0.15 \\
0.15
\end{array}\right], \quad E^{t}(2)=\left[\begin{array}{l}
0.3 \\
0.4 \\
0.3
\end{array}\right] .
\end{aligned}
$$

Mode 3.

$$
\begin{aligned}
A(3) & =\left[\begin{array}{ccc}
0.25 & 0.15 & -0.1 \\
0.2 & -0.6 & 0.1 \\
-0.1 & 0.3 & 0.5
\end{array}\right], \quad C(3)=\left[\begin{array}{ll}
1 & 0 \\
0 & 1 \\
0 & 0
\end{array}\right], \\
H_{1}(3) & =\left[\begin{array}{c}
0.1 \\
0.15 \\
0.2
\end{array}\right], \quad E^{t}(3)=\left[\begin{array}{l}
0.2 \\
0.4 \\
0.5
\end{array}\right] .
\end{aligned}
$$

For the three modes, we use $\mathscr{W}=1.2 I, \mathscr{V}=0.6 I$, and $R_{o}=0.15 I$. Numerical computations of (3.1) and (3.2) using Matlab-LMI solver are summarized in Table 4.1.

For the purpose of comparison, Table 4.2 gives the associated costs of both the guaranteed cost filter designed for the nominal system and the optimal filter developed in this paper. It is clear that the latter outperforms the standard one in the presence of parametric uncertainty. 
Table 4.1. Summary of computational results.

\begin{tabular}{c|c|rr|rrr}
\hline Mode & Cost & \multicolumn{2}{|c|}{$G_{i} \times 10^{6}$} & \multicolumn{3}{c}{$F_{i}$} \\
\hline \multirow{3}{*}{1} & \multirow{3}{*}{$112.731 \times 10^{-4}$} & -0.866 & -1.322 & 0.351 & -0.107 & -0.041 \\
& & 7.611 & 6.819 & -0.707 & 0.413 & 0.159 \\
& & -0.923 & 12.775 & -1.582 & -1.027 & 0.961 \\
\hline \multirow{3}{*}{2} & \multirow{3}{*}{$185.236 \times 10^{-4}$} & 5.732 & -2.289 & -0.102 & -0.196 & 0.221 \\
& & 10.345 & 12.701 & 0.245 & -0.722 & 0.651 \\
\hline \multirow{3}{*}{3} & \multirow{3}{*}{$147.246 \times 10^{-4}$} & -0.765 & 1.545 & -0.282 & 0.049 & -0.124 \\
& & 2.498 & 12.763 & 0.176 & -0.411 & -0.017 \\
& & -3.127 & 4.161 & 0.484 & 0.756 & -0.621 \\
\hline
\end{tabular}

Table 4.2. A cost comparison between standard and optimal QGC filters.

\begin{tabular}{lccc}
\hline Filter & $\Delta_{k}=-0.8$ & $\Delta_{k}=0$ & $\Delta_{k}=0.8$ \\
\hline Standard & $63.732 \times 10^{-2}$ & $31.194 \times 10^{-2}$ & $226.572 \times 10^{-2}$ \\
Robust & $112.554 \times 10^{-4}$ & $137.093 \times 10^{-4}$ & $148.632 \times 10^{-4}$ \\
\hline
\end{tabular}

\section{Conclusions}

In this paper, the problem of the design of robust steady-state estimator for a class of uncertain discrete-time systems with Markovian jump parameters has been addressed. The results obtained here have extended the normal steady-state Kalman filter to the case of systems with norm-bounded time-varying uncertainties in the state and measurement equations as well as jumping parameters. A linear state estimator has been constructed such that the estimation error covariance is guaranteed to lie within a certain bound for all admissible uncertainties. The solution has been given in terms of a family of linear matrix inequalities.

\section{Acknowledgment}

This work was supported by the Scientific Research Council of the United Arab Emirates University under Grant no. 02-04-7-11/03. It was also partially supported by the Laboratory of Complex Systems and Intelligence Science, Institute of Automation, Chinese Academy of Sciences, China.

\section{References}

[1] B. D. O. Anderson and J. B. Moore, Optimal Filtering, Prentice-Hall, New Jersey, 1979.

[2] M. Athans, Command and control (C2) theory: a challenge to control science, IEEE Trans. Automat. Control 32 (1987), no. 4, 286-293.

[3] D. S. Bernstein and W. M. Haddad, Steady-state Kalman filtering with an $\mathrm{H}_{\infty}$ error bound, Systems Control Lett. 12 (1989), no. 1, 9-16.

[4] E.-K. Boukas and A. Haurie, Manufacturing flow control and preventive maintenance: a stochastic control approach, IEEE Trans. Automat. Control 35 (1990), no. 9, 1024-1031. 
[5] E.-K. Boukas, Q. Zhang, and G. Yin, Robust production and maintenance planning in stochastic manufacturing systems, IEEE Trans. Automat. Control 40 (1995), no. 6, 1098-1102.

[6] O. L. V. Costa and M. D. Fragoso, Stability results for discrete-time linear systems with Markovian jumping parameters, J. Math. Anal. Appl. 179 (1993), no. 1, 154-178.

[7] _ Discrete-time LQ-optimal control problems for infinite Markov jump parameter systems, IEEE Trans. Automat. Control 40 (1995), no. 12, 2076-2088.

[8] C. E. De Souza and M. D. Fragoso, $H^{\infty}$ control for linear systems with Markovian jumping parameters, Control Theory Adv. Tech. 9 (1993), no. 2, 457-466.

[9] $\quad H_{\infty}$ filtering for Markovian jump linear systems, Proc. 35th IEEE Conf. on Decision \& Control, IEEE, New York, 1996, pp. 4814-4818.

[10] - Robust $H_{\infty}$ filtering for uncertain Markovian jump linear systems, Proc. 35th IEEE Conf. on Decision \& Control, IEEE, New York, 1996, pp. 4808-4813.

[11] F. Dufour and P. Bertrand, The filtering problem for continuous-time linear systems with Markovian switching coefficients, Systems Control Lett. 23 (1994), no. 6, 453-461.

[12] _ An image-based filter for discrete-time Markovian jump linear systems, Automatica J. IFAC 32 (1996), no. 2, 241-247.

[13] Y. Ji, H. J. Chizeck, X. Feng, and K. A. Loparo, Stability and control of discrete-time jump linear systems, Control Theory Adv. Tech. 7 (1991), no. 2, 247-270.

[14] H. J. Kushner, Stochastic Stability and Control, Mathematics in Science and Engineering, vol. 33, Academic Press, New York, 1967.

[15] M. S. Mahmoud, Robust performance results for discrete-time systems, Math. Probl. Eng. 3 (1998), no. 6, 517-538.

[16]_ Robust Control and Filtering for Time-Delay Systems, Control Engineering, vol. 5, Marcel Dekker, New York, 2000.

[17] M. S. Mahmoud, L. H. Xie, and Y. C. Soh, Robust Kalman filtering for discrete state-delay systems, IEE Proc. Control Theory Appl. 147 (2000), 613-618.

[18] D. D. Moerder, N. Halyo, J. R. Broussard, and A. K. Caglayan, Application of precomputed control laws in a reconfigurable aircraft flight control system, J. Guidance Control Dynam. 12 (1989), no. 3, 325-333.

[19] T. Morozan, Stability and control for linear systems with jump Markov perturbations, Stochastic Anal. Appl. 13 (1995), no. 1, 91-110.

[20] I. R. Petersen and D. C. McFarlane, Robust state estimation for uncertain systems, Proc. 30th IEEE Conf. Decision \& Control, IEEE, New York, 1991.

[21] D. B. Petkovski, Multivariable control system design: a case study of robust control of nuclear power plants, Fault Detection and Reliability 9 (1987), 239-246.

[22] P. Shi, Filtering on sampled-data systems with parametric uncertainty, IEEE Trans. Automat. Control 43 (1998), no. 7, 1022-1027.

[23] P. Shi and E.-K. Boukas, $H_{\infty}$-control for Markovian jumping linear systems with parametric uncertainty, J. Optim. Theory Appl. 95 (1997), no. 1, 75-99.

[24] P. Shi, E.-K. Boukas, and R. K. Agarwal, Control of Markovian jump discrete-time systems with norm bounded uncertainty and unknown delay, IEEE Trans. Automat. Control 44 (1999), no. 11, 2139-2144.

[25] _ Kalman filtering for continuous-time uncertain systems with Markovian jumping parameters, IEEE Trans. Automat. Control 44 (1999), no. 8, 1592-1597.

[26] P. Shi, C. E. De Souza, and L. Xie, Robust $H_{\infty}$ filtering for uncertain systems with sampled-data measurements, Proc. 32nd IEEE Conf. Decision \& Control, IEEE, New York, 1993, pp. $793-$ 798.

[27] D. D. Sworder and R. O. Rogers, An LQ-solution to a control problem associated with a solar thermal central receiver, IEEE Trans. Automat. Control 28 (1983), no. 8, 971-978. 
[28] A. S. Willsky, A survey of design methods for failure detection in dynamic systems, Automatica J. IFAC 12 (1976), no. 6, 601-611.

[29] W. M. Wonham, Random differential equations in control theory, Probabilistic Methods in Applied Mathematics, Vol. 2 (A. T. Bharucha-Reid, ed.), Academic Press, New York, 1970, pp. 131-212.

[30] L. Xie, C. E. De Souza, and M. D. Fragoso, $H_{\infty}$ filtering for linear periodic systems with parameter uncertainty, Systems Control Lett. 17 (1991), no. 5, 343-350.

[31] L. Xie and Y. C. Soh, Robust Kalman filtering for uncertain systems, Systems Control Lett. 22 (1994), no. 2, 123-129.

[32] L. Xie, Y. C. Soh, and C. E. De Souza, Robust Kalman filtering for uncertain discrete-time systems, IEEE Trans. Automat. Control 39 (1994), no. 6, 1310-1314.

Magdi S. Mahmoud: College of Engineering, United Arab Emirates University, P.O. Box 17555, Al-Ain, United Arab Emirates

E-mail address: magdism@uaeu.ac.ae

Peng Shi: School of Technology, University of Glamorgan, Pontypridd, Wales, CF37 1DL, UK

E-mail address: pshi@glam.ac.uk 


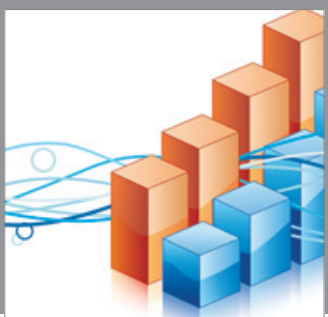

Advances in

Operations Research

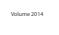

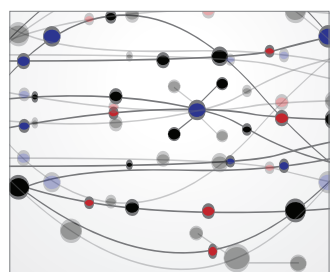

\section{The Scientific} World Journal
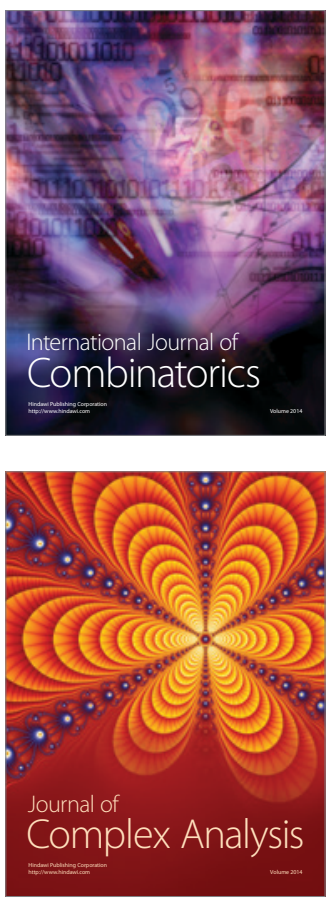

International Journal of

Mathematics and

Mathematical

Sciences
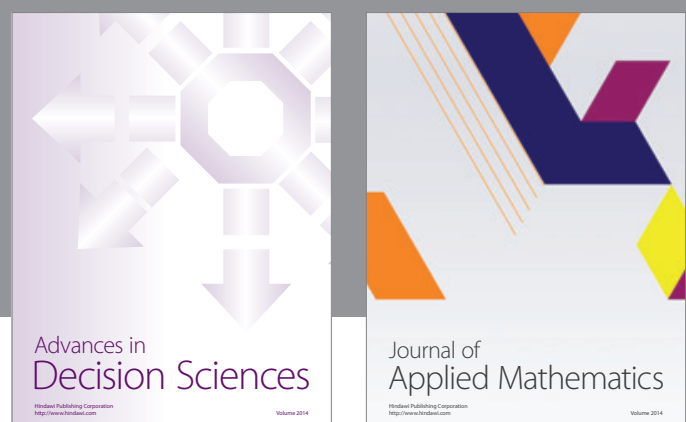

Journal of

Applied Mathematics
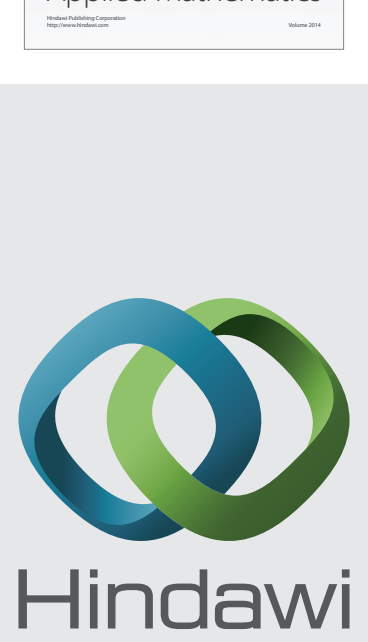

Submit your manuscripts at http://www.hindawi.com
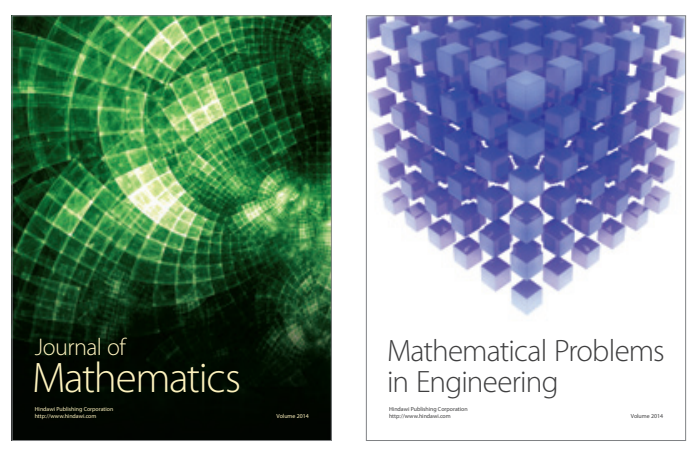

Mathematical Problems in Engineering
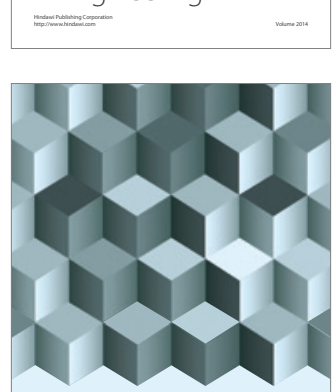

Journal of

Function Spaces
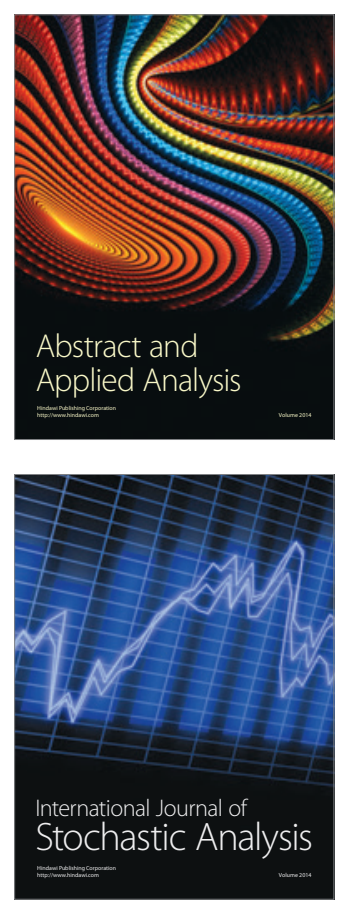

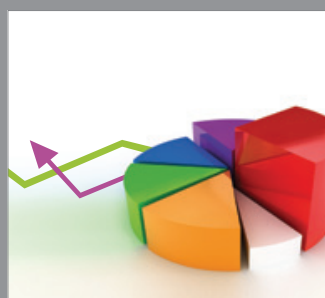

ournal of

Probability and Statistics

Promensencen
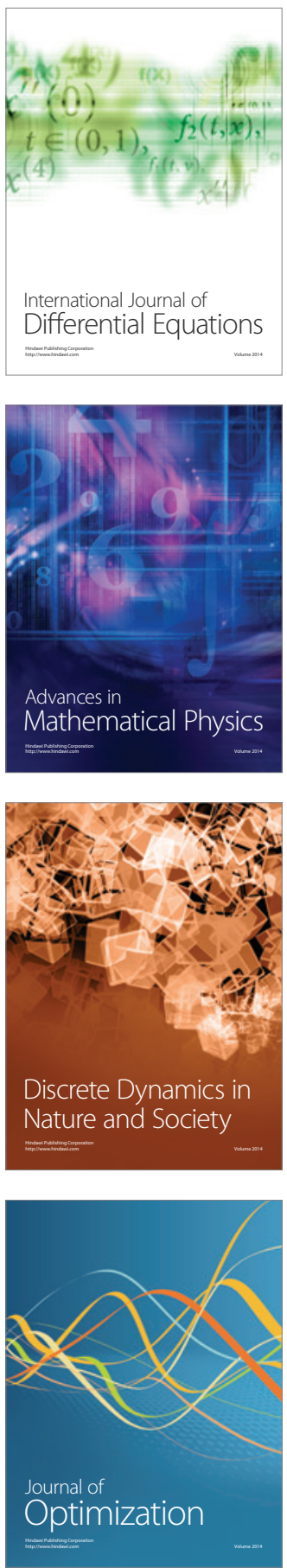